\section{Bus Systems For Process Control}

\author{
R.J. Rausch \\ CERN, Geneva, Switzerland \\ Associate Member of EPS
}

Buses used in large experimental facilities to communicate between elements of control systems have strongly influenced the architecture of these systems over the past 20 years. The buses were initially employed in conjunction with minicomputers for data acquisition and control applications: today they support a variety of high-speed processors and powerful control modules on a single entity - the data highway.

The standardization of data highways became necessary when physicists and engineers realized that general purpose functions could be implemented as standard modules plugged onto an agreed dataway. Teams from different laboratories could then collaborate more efficiently on large experiments, with each team contributing its know-how and using compatible material to assemble larger data acquisition and control systems.

Complex performance features, new applications and alternative approaches to bus systems have emerged following the early successes of CAMAC, the first wideIy used parallel bus system. In helping a user select the optimum system from among a profusion of commercial products, it is useful to consider the impact on control system architectures of Europe's three most popular parallel bus systems (CAMAC, G64 and VMEbus).

\section{CAMAC}

The standardized bus systems adventure started in 1965-66 when European high energy physics laboratories established the CAMAC system definition [1].

Despite its early specification (in 1969), CAMAC equipment is being produced around the world and is still widely used for data acquisition. CAMAC's major advantages are flexibility to build optimized systems and the interchangeability of commercial equipment. Existing control systems can thus be easily restructured and upgraded by incorporating more powerful modules. It also provides independence from computer manufacturers in that the large capital investment in CAMAC for control systems is not lost when obsolete computers are replaced.

Moreover, CAMAC is a standard bus system, developed "by users for users",

Raymond Rausch studied physics and electronic engineering at the Conservatoire National des Arts et Métiers, Paris. He has been an accelerator controls engineer with CERN, Geneva since 1972 after setting up data acquisition systems at the linear accelerator at the CNRS, Orsay. which has adapted technical advances to meet complex requirements.

\section{Parallel highway}

CAMAC initially handled the input/output $(\mathrm{I} / \mathrm{O})$ needs of the computer to which it was connected via an electronic interface that matched the computer's internal bus to the electrical specification of the CAMAC dataway. The EUR- 4100 specification thus describes a bus system comprising a single master, or controller, and up to 23 slave modules in a crate with 25 slots for circuit boards. The master acts as an interface module between the computer's $\mathrm{I} / \mathrm{O}$ bus and the slave modules. Many different CAMAC slave modules are available on the market (e.g. simple $1 / 0$ or status registers to coincidence selectors and triggers for complicated, high-speed events).

The dataway is a parallel highway with 24 read lines from modules to the controller and 24 write lines in the opposite direction. A station address, a sub-address and a function code constitute the CAMAC command. Two dedicated signal lines connect each station to the controller: one addresses the station (either alone or in combination with other stations) and the module uses the other to inform the controller that it requires attention - a very useful interrupt capability.

\section{Branch highway}

A single CAMAC crate connected to a computer soon became insufficient and the relatively high price of minicomputers justified the simultaneous control of several CAMAC crates. One could either connect several computer-dedicated crate controllers to the $\mathrm{I} / \mathrm{O}$ bus (if this was electrically possible) or use a single interface between the computer and a standard parallel bus onto which several CAMAC crates were branched. A second CAMAC specification (EUR-4600) describes a common vertical bus, the CAMAC branch highway $(\mathrm{BH})$ linking seven slave CAMAC crates to a single computer. A BH driver interface for each major type of computer was then developed to ensure independence from manufacturers of electronic circuits.

\section{Serial highway}

A CAMAC serial highway has been specified (EUR-6100) to extend the crate addressing capability and the bus length. It has the form of a loop starting from the master controller, connecting up to 62 crates and returning to the controller. Compared with the branch highway, its physical structure is simplified but the protocol is more complex and includes powerful error detection features.

\section{Multiple controllers}

The last improvement to the CAMAC system was made in 1979 to allow more than one controller in each CAMAC crate, while retaining full compatibility with existing modules. Two or more dataway masters could operate in a crate, and bus mastership protocols had to be specified to ensure orderly sharing of the dataway. This new capability (EUR-6500) allowed computing capacity to increase to solve critical real-time problems by exporting them out of the computer into the CAMAC crate.

\section{Microprocessors}

The introduction of microprocessors after the original CAMAC specifications were published has greatly enhanced the versatility and performance of CAMAC equipment. A microprocessor can be used in a module to pre-process data or to handle a communication protocol between the module and some external device. It can also be located in the crate controller used for sequencing and handling data in a simple stand-alone system; or in a crate which is also connected to a parallel or serial highway to perform local control or processing and so relieve the loads on the computer and on the highway. Finally, the microprocessor can be located in a separate controller module called an auxiliary crate controller (ACC) to act like a local controller or processor.

\section{8-Bit Microprocessor Buses}

The emergence of 8-bit microprocessors in 1978-79 gave rise to a demand for inexpensive, compact, intelligent buses with CAMAC's modularity in applications where CAMAC equipment was not present and where a few $\mathrm{I} / \mathrm{O}$ signals called for some local processing. Very simple microprocessor buses, usually optimized for a given type of microprocessor, appeared on the market.

Several groups in the early 1980's selected the $\mathrm{G} 64$ bus system from GESPAC for applications at CERN's LEP accelerator. It involves a single height Eurocard format $(100 \mathrm{~mm} \times 160 \mathrm{~mm})$ for the printed circuit boards and a crate containing a maximum of 10 cards. The dataway uses 64 signal lines to provide 18 address lines, 16 data lines, one interrupt line and memory cycles for the microprocessors. Direct memory access (DMA) transfers are also specified.

The basic G64 specification has since been enhanced to cater for new technical needs. The number of cards per crate has been extended to 16 by changing the dataway line drivers, and a double height Eurocard was introduced together with a front panel to facilitate attaching $\mathrm{I} / \mathrm{O}$ cable connectors. 
More than $2000 \mathrm{G} 64$ bus systems are used in LEP. Industrial sub-systems are connected to the LEP control system via a G64 crate in which the microprocessor acts as a communications protocol converter. The various systems are closely linked to the equipment they control so they can provide autonomous control sequences, the surveillance and monitoring of sub-systems and alarm generation.

Hierarchically, the G64 systems are connected to process control assemblies (PCA's) that actually drive beam line equipment via buses (MIL-STD-1553-B protocol). It is also at the level of the G64 bus systems that the real-time operation and synchronization of controls arise.

The G64 microprocessors mostly use a simple software kernel and are programmed in PASCAL or BASIC languages. The addressing capability (64 Kbyte) of the microprocessor used in G64 is sufficient for most simple applications. More CPU power and memory to implement more complex functions and the need for standard real-time software kernels (see page 34) means that 8-bit systems are reaching their limits: 16 - or even 32 -bit bus systems will be required.

\section{VMEbus}

The first formal specification of the VMEbus appeared in 1982 (Revision A of the IEEE 1014 specification). Owing to its functional modules, VMEbus is essentially a Eurocard version of Motorola's Versabus, hence "Versabus Modèle Européen" (VME). VMEbus has been improved and extended into a three-bus combination VME-VSB-VMS). VSB is a fast multipro-

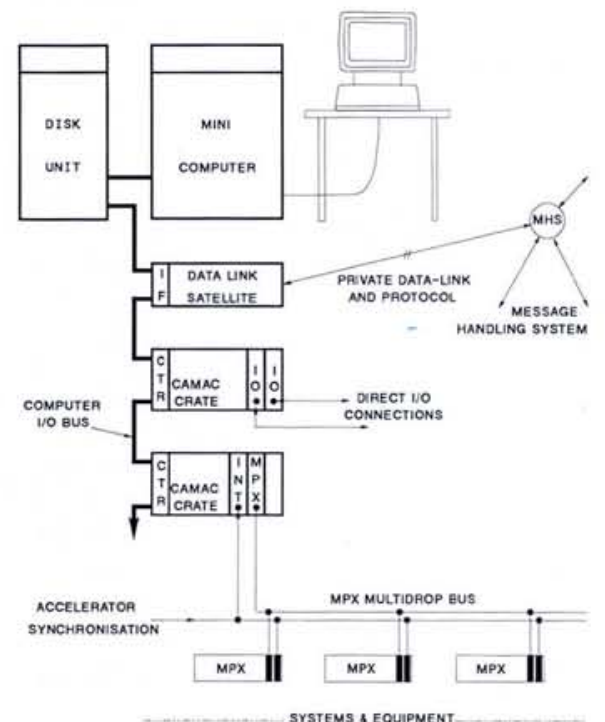

cessor and memory sub-bus; VMS provides a serial connection between modules for communications between processors.

The VMEbus specification aimed to define a dataway to interconnect data processing, data storage, peripheral control devices and network interfaces into a closely coupled configuration. It is also intended to allow multiprocessor configurations capable of handling data and address paths of up to 32 bits, and to adapt the Eurocard sub-rack, an existing and widely used mechanical standard.

The essential VMEbus feature is a multiprocessor bus with 2 to 21 slots for Eurocards (single or double height). The connector of the single height card provides 16-bit data, 24-bit address, 7 interrupt lines and all the control lines. The second connector available on the double height card adds additional 16-bit data and 8-bit address. The additional 64 pins on this second connector are dedicated to the VSB extension. VSB is limited to a maximum of six slots to ensure high-speed data transfer between up to six modules and a mechanism to arbitrate amongst processors requesting mastership is provided.

VMEbus contains all the necessary features typically required for operating multiprocessor systems: it is widely recognized and is supported by more than 200 manufacturers.

\section{Impact On Control Architectures}

The impact of bus systems on control architectures can be evaluated by comparing CERN's SPS and LEP control systems that were designed in $1972-76$ and in 1983-86, respectively.

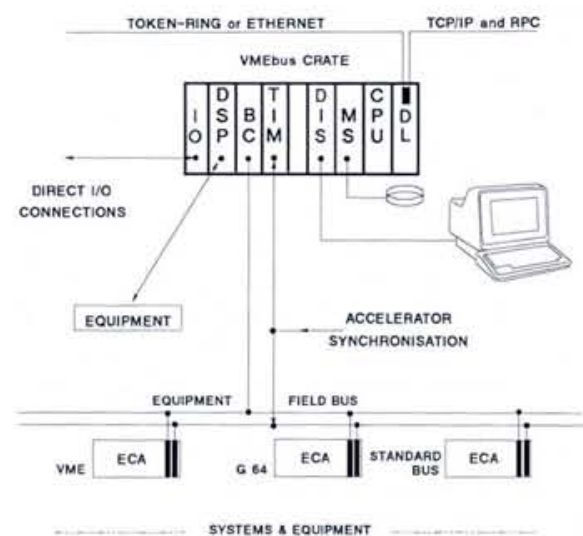

Fig. 1 - Control architectures for CERN accelerators. (a - left) Super Proton Synchrotron (SPS) designed in the mid-1970's. CAMAC crates with slotted circuit boards are interfaced either by CAMAC branch and serial highways or through dedicated crate controllers to link the accelerator's devices to minicomputers distributed over the $7 \mathrm{~km}$ in circumference site. A multidrop bus and lines for timing signals drive equipment via MPX modules (MPX is a CERN internal standard).

(b - right) Large Electron Positron Collider (LEP) designed in the mid-1980's. Control functions (TIM: timing, BC: field bus connection, etc.) are provided by microprocessor boards grouped together in a single VMEbus crate. The equipment field bus and the synchronization signal lines constitute local distribution networks to electronic control assemblies (ECA) that drive devices. Distributed intelligence is linked by token ring or Ethernet networks.

\section{SPS}

The SPS control system (Fig. 1a) is based on minicomputers, a proprietary real-time kernel and a software development system which became available in the early 1970's. A system connecting numerous minicomputers distributed over the SPS site (7 $\mathrm{km}$ in circumference) had to be developed, even though a standard network protocol had not been specified and the accelerator's control hardware was not yet available.

The interface to the accelerator equipment was implemented in CAMAC, then the only international standard. CAMAC crates were interfaced either directly to the computer $\mathrm{I} / \mathrm{O}$ bus via dedicated crate controllers or by using the standard CAMAC branch and serial highways with associated crate controllers.

Field buses for equipment were not available so an economic serial bus with crates and electronic modules had to be developed to connect many equipment crates to the local computer. This is the CERN SPS/MPX system with more than 700 crates and 6000 modules which are still in operation.

\section{LEP}

LEP'S control system was drawn up when communication network standards and multiprocessor bus systems were being specified and when 16-bit microprocessors with a large addressing capability became commercially available [2].

Owing to the rapid evolution of integrated circuit technology and the expansion of the multiprocessor features of the VMEbus system, all the process control functions could be grouped into separate VMEbus multiprocessor crates (see Fig. 1b). Each crate is connected to a local or a wide area network conforming to international standards (Ethernet IEEE 802.3 or token ring IEEE 802.5) using a data link module. The network protocol uses the widely recognized TCP/IP standard together with remote procedure calls (RPC) for which an international standard is being finalized.

The central processor unit is based on a 32-bit microprocessor with up to 8 Mbyte of memory. It runs under a real-time operating system (currently OS-9) on which the RPC is implemented. Local interaction with the processor is done via a simple terminal linked by a RS-232-C standard cable. A display module can be inserted into the VMEbus to give locally available high resolution graphics combined with an interactive touch terminal. Local mass storage of data on a hard disk is achieved with a plug-in unit interfaced either directIy to the VMEbus or to the main processor unit via a standard SASI or SCSI bus connection system.

For rapid data acquisition and the transfer of large amounts of data, e.g. output from beam instrumentation, specialized I/O modules can be plugged-in and linked 
directly to detectors. Some demultiplexing is acceptable in less demanding applications where many similar items of equipment are controlled, e.g. power supplies. Here, a field bus controller drives a multidrop cable connecting up to 30 equipment control assemblies (ECA's) implemented using the MIL-STD-1553-B standard field bus. The ECA's, located close to the subsystems they control, consist of G64 or VMEbus crates with microprocessors.

\section{Concluding Remarks}

Standardization of bus systems has allowed investments in design, material, manpower and technical know-how to be capitalized upon. Meanwhile, the diversity of applications, users' experience and contributions to international standards bodies have enhanced and optimized technical specifications.

One consequence is a change-over from the use of buses for simple input/output functions to the complete integration of all the operations required for data acquisition and process control. However, comparisons between bus systems used for controlling large accelerators suggest that the basic requirements have not changed drastically in 20 years: distributed communication, local processing power, remote control and surveillance, and local interaction with graphics remain essential.

The revolution is that these functions are now implemented more quickly, economically and flexibly, and with greater pre-processing of data. The integration of complete functions in a single chip (i.e. network protocols, processors, memories, digital signal processors, peripheral controllers, intelligent sensors, etc.) and the standardization of hardware for bus systems means that more computer power and processing functions can now be packed into a single multiprocessor VMEbus crate than in a complete 1970's style front-end process control assembly requiring several racks of equipment.

Further improvements in software, standardization of software tools, languages, real-time kernels, communication packages, graphics packages, etc. are essential to improve the efficiency of process control. For instance, we dream of software modules plugging into a standard software system (the "software bus") much like a VMEbus module plugs into a standard VMEbus. Moreover, we are already not far from a UNIX real-time software kernel capable of dynamic load-sharing among several identical VMEbus processors in a crate and remotely communicating with user-friendly workstations for the operators.

\section{REFERENCES}

[1] EUR 11079: Guide to Internationally Recognized Hardware Interfaces (CEC, Brussels) 1988.

[2] Altaber J. et al., Proc.1983 Particle Accelerators Conference, IEEE Trans. NS-30 (1983).

\section{CIM For Particle Factories}

Large experimental facilities may seem unusual to the average person, but they are comparable to industrial machines suggesting an industrial approach for integrating computers. A major installation such as an accelerator complex or "particle factory" is mostly made up of well-defined industrial components so its operation largely leans on functional blocks similar to those found in industry.

But this is not the generally accepted view. The problems of pioneering in statistics-based research topics such as particle physics lead engineers and physicists to work at the limits of what is possible in several fields (e.g. electronics, mechanics, computing, materials, etc.). They are forced to permanently explore novel ideas and to evaluate and apply promising new technologies as soon as they emerge from laboratories. This feature also pushes them into initiating new developments until they begin to feel they have to step beyond in dustry. One finally becomes accustomed to developing everything that is needed be cause one generally achieves better results than with what is easily available.

This has been the case on introducing computers into experimental facilities. There has been an anarchic development of tools for engineering, production, control, planning and administrative support activities. Isolation from developments elsewhere and the lack of defined goals rapidly became the main problems. The software industry was in the meantime developing products for a much larger community.

\section{Exploiting CIM}

The spectacular progress of informatics has generated considerable interest in attempts to model a commercial enterprise. Views of an experimental facility, notably a particle factory, that are developed using some of the latest descriptions [1, 2] emphasize two unique features:

- the facility does not sell, strictly speaking, the products it manufactures;

- products such as high energy particles cannot be stored or transported in a conventional way as these operations need complex machines. The products are instead manufactured for local use by physicists.

Considering an experimental facility as a manufacturing site nonetheless provides a working framework for achieving greater efficiency. A computer-integrated manufacturing (CIM) system should thus be considered for an experimental facility as reports show that its implementation produces encouraging results $[3,4]$ by generating major improvements in productivity, cost savings, throughput and flow control.

Although CIM is not yet clearly defined [3] it aims to integrate computers and adequate software tools, which may not necessarily exist as yet, into all aspects, including management, of a manufacturing cycle (planning, design, etc.). A top-down approach to implementing CIM leans on two fundamental pillars: communication and data exchange and management. Success requires:

- A coherent and powerful communication network based on international standards that links together all laboratories, offices, workshops, accelerators and experiments. Organized into interconnected islands of local area networks (LAN's), the overall network allows for an independent and flexible selection of computer-based products aimed at helping workers.

- Computer-based software tools that comply fully with international data exchange standards.

- A single, widely accessible database management system (DBMS) for the collection and retrieval of data originating from different sources (administrative, technical, etc.) or having different natures (text, picture, sound, etc.).

Weaknesses in any of these areas result in isolated islands of different computerbased tools to aid the different groups (systems designers, operators, etc.) and a large reduction in the overall benefits. A correctly configured CIM system can help by acting as the glue between individual tools used for different functions in a company [5].

CERN is an example of one research organization that is currently working towards integration. A powerful communication network has been implemented, with Ethernet and token rings as wide area networks and with Ethernet and communication systems based on the MIL-1553-B standard for LAN's. A DBMS has been approved for both process control and administrative activities. Software tools are starting to be connected and although links are missing (mainly in administrative and support areas), an ambitious project to integrate all administrative tasks is underway.

\section{Conclusions}

Large experimental facilities in physics have enjoyed the advantage of an intimate contact with computers. But the complex, slightly anarchic situation which grew up should not divert attention from the coherence that is now emerging elsewhere. It is essential that managers of these centres should now seek this coherence when approving computer-based software products. The application of full integration based on the CIM top-down approach applied to an experimental facility could then serve as a model for European industry.

\section{REFERENCES}

[1] Kosankc K. and Vlietstra J., Proc. 6th Ann. ESPRIT Conf., Brussels (1989).

[2] Playoust P., Tech. de I'Ingénieur, H6050. [3] Foltz M.T. and Tanik N.M., Int. J. Computer Applns. in Technology 3 (1990) 26.

[4] Costea I., Information Strategy, Summer issue (1990).

[5] Leonard R., Int. J. Computer Integrated Manufacturing I (1990) 13.

M. Rabany CERN, Geneva 